\section{Autogestión del Gobierno Autónomo descentralizado municipal de Pangua y su repercusión en la prestación de servicios}

The research focuses on self-management in the Autonomous Decentralized Pangua Government of the province of Cotopaxi

\section{Patricia Genovezzy Velásquez \\ Roger Yela Burgos* \\ Liliana Napa* \\ Alexandra Isabel Cardenas Loor*}

\section{RESUMEN}

La investigación versa sobre la Autogestión en el Gobierno Autónomo Descentralizado de Pangua de la provincia de Cotopaxi, como herramienta eficaz probada que exalta la utilización de los mejores valores del individuo y de los grupos, situándolos en mejor posición para enfrentar y resolver sus problemas comunes. El principal objetivo de esta investigación es evaluar la efectividad de la autogestión en la prestación de servicios del Gobierno Autónomo Descentralizado Municipal de Pangua. Con esta investigación sirve como aporte al procedimiento de

\footnotetext{
* Ingeniera en Administración de Empresas, Facultad de Ciencias Empresariales , Universidad Técnica Estatal de Quevedo, QuevedoEcuador. pattyrocig76@gmail.com; https://orcid.org/0000-0002-85565777

* Economísta, Facultad de Ciencias Empresariales, Universidad Técnica Estatal de Quevedo, Quevedo-Ecuador. ryela@uteq.edu.ec, https://orcid.org/0000-0003-0643-163X

* Contador Público Autorizada, Facultad de Ciencias Empresariales, Universidad Técnica Estatal de Quevedo, Quevedo-Ecuador.

filiylili84@gmail.com; https://orcid.org/0000-0002-5652-34I2

* Licenciada en Educación con mención educación infantil Universidad Particular de Loja, Quevedo. Ecuador. alexandracardenasadonai@gmail.com; https://orcid.org/0000-0002$979 \mid-3679$
}

\section{REVISTATECNOLÓGICA ciencia y educación Edwards Deming}

ISSN: 2600-5867

\section{Atribución/Reconocimiento-NoCo} mercial- Compartirlgual 4.0 Licencia Pública Internacional — CC

\section{BY-NC-SA 4.0}

https://creativecommons.org/licenses /by-nc- sa/4.0/legalcode.es

Editado por: Tecnológico Superior Corporativo Edwards Deming Julio - Diciembre Vol. 5 - 2 - 202 I https://revista-edwardsdeming.com/index.php/es e-ISSN: 2576-097I

Recibido: 10 junio 2020

Aprobado: 4 Enero, 202I

Pag 32-39 
diseño y establecimientos de indicadores de autogestión de eficacia y eficiencia para evaluar la efectividad de la autogestión en la prestación de servicios, dentro o entre distintas áreas del Municipio herramientas fáciles de utilización que le permitan un desarrollo integral sustentable y sostenible a través del tiempo, con una planeación estratégica integral. Se tomó como base a los empleados usuarios del Gobierno Autónomo Descentralizado Municipal de Pangua a los cuales se realizará la encuesta y entrevista personalizada, donde las respuestas nos ayudó a llegar al objetivo deseado.

Palabras clave: Autogestión; Autónomo, Descentralizado, Gobierno

\section{ABSTRACT}

The research focuses on self-management in the Autonomous Decentralized Pangua Government of the province of Cotopaxi, as proven effective tool that enhances the use of the best values of individuals and groups, placing them in a better position to face and solve their common problems. The main objective of this research is to evaluate the effectiveness of self-management in the provision of services Decentralized Autonomous Municipal Government of Pangua. With the investigation is expected to contribute to the process of design and local self-management indicators of effectiveness and efficiency to evaluate the effectiveness of self-management in the provision of services within or between different areas of the municipality easy to use tools enable a sustainable and comprehensive sustainable development over time, with a comprehensive strategic planning. It shall be based employees and users of the Autonomous Decentralized Municipal Government Pangua to which the survey and personal interview, where responses will help us to reach the desired goal is made.

Keywords: Self-management; Autonomous, Decentralized, Government

\section{INTRODUCCIÓN}

La autogestión es un proceso integral de fortalecimiento organizativo, secuencia en las acciones y una capacitación continua, que es robustecer las propias capacidades antes, durante y después de la entrega del bien adquirido, descubriendo las capacidades, destrezas, habilidades individuales, directivas y de grupo de la población. La autogestión comunitaria se conduce con autonomía en coordinación con los intereses y acciones con otros grupos. Ya que este proceso se genera al romper la dependencia y marginación previas para crear un espacio propio de representación, defensa y coordinación, la autogestión conduce necesariamente a la conquista gradual de poder económico, social y político. 
La autogestión comienza, se desarrolla y culmina transformando la actitud, disposición de los miembros de la comunidad hacia sí mismos y hacia los demás. La autogestión no debe ser un episodio que brille fugazmente en la vida de una comunidad para luego desaparecer, la idea es lograr cambios que sean permanentes, por ello es imprescindible el proceso de sustentabilidad, para ello planteamos dos formas:

Por un lado, debemos preservar el ecosistema, garantizando los recursos naturales a las generaciones futuras. Por otra parte, el proceso debe ir ganando una estabilidad cada vez mayor, a fin de asegurar su continuidad, ya que la comunidad debe tener una imagen de sustentabilidad de los proyectos a ellos entregados, para poder negociar en el futuro con el resto de la sociedad u otras instituciones otro tipo de apoyo. Resultado que ha de ser el logro de la coordinación y el poder necesarios para proponer e impulsar la descentralización del Estado, a través de contar con una visión y misión para llenar los vacíos que las instituciones relacionadas no pueden acceder a este tipo de necesidades comunitarias.

La autogestión cuenta para su implementación con la Planificación alternativa, aspecto práctico del trabajo comunitario. La autogestión comunitaria, como práctica social, es un sistema de planificación alternativo que opera en la propia comunidad, poniendo en práctica actividades conjuntas en torno a intereses compartidos implica conocer la realidad desear un cambio positivo, idear un futuro mejor; definir las acciones necesarias para alcanzar esas metas definiendo el camino que facilita lo deseado para construir la organización adecuada que haga realidad esta meta.

La presente investigación tiene el carácter de explicativa, porque va más allá de la descripción de conceptos o el establecimiento de relaciones. Está dirigida a responder las causas de los eventos de autogestión, pretendiendo responder preguntas como; ¿En qué nivel considera usted que es efectiva la autogestión del Gobierno Autónomo Descentralizado de Pangua en la prestación de servicios municipales? ¿Conoce si se están realizando proyectos de Autogestión? Por lo tanto, este tipo de investigación será estructurada, lo que implica realizar exploraciones y descripciones de manera complementaria.

\section{MATERIALES Y MÉTODOS}

El presente estudio pretendió describir para luego analizar sus componentes. Se empleó también las técnicas de investigación directa, que consiste en visitas y trabajo de campo, para lo cual se realizaron encuestas que fueron procesadas y sintetizadas como soporte de la investigación de acuerdo a los temas específicos tratados.

Para la realización de esta investigación se recurrió a la aplicación de los siguientes métodos: 
Este método ayudó a plantear la problemática que se investiga; autogestión del Gobierno autónomo municipal de Pangua y su incidencia en la prestación de servicios. Por medio de este método se pudo, estructurar y desarrollar el marco teórico: como son: teorías y principios en que se fundamenta la investigación. Mediante este método se pudo realizar el análisis de la información obtenida en el proceso de investigación para establecer resultados; y también formular las respectivas conclusiones y recomendaciones. Este método condujo al planteamiento coherente y pertinente de una propuesta alternativa que genere transformaciones relevantes en el gobierno autónomo municipal, institución donde se realizó la investigación.

Las técnicas de investigación son mecanismos encargados de recolectar, sistematizar, procesar, almacenar información y datos para la demostración de la hipótesis, que se aplicó a los empleados/as del Gobierno Autónomo descentralizado municipal de Pangua. Las técnicas e instrumentos utilizados permitieron recolectar la información para el análisis empírico, el mismo que será confrontado con el marco teórico del proyecto de investigación.

Las técnicas empleadas en la medición de las variables de investigación fueron:

Observación consistió en la percepción sistemática y estuvo dirigida a captar los aspectos más significativos de los objetos, hechos, realidades sociales y personas, de la institución. Proporcionando la información empírica necesaria para plantear la investigación.

Entrevistas fue un diálogo solicitado de manera personal con los directivos de la institución, con el propósito de obtener información.

La encuesta estuvo destinada a recopilar información a través de un cuestionario que fue un conjunto de preguntas, preparado cuidadosamente, sobre los hechos aspectos que interesaban en la investigación, para que sea contestado por los empleados y ciudadanos de la muestra.

Se utilizó, además, una técnica de investigación bibliográfica, destinada a obtener información de fuentes secundarias que constan en libros, revistas, periódicos y documentos en general; la misma que fue la técnica de análisis de documentos. aspectos que interesaban en la investigación, para que sea contestado por los empleados y ciudadanos de la muestra.

La población del cantón Pangua, usuaria externa del GOBIERNO AUTÓNOMO DESCENTRALIZADO MUNICIPAL DE PANGUA se estimó en 8.427 habitantes al extrapolarse desde el año $200 \mathrm{I}$ hasta el año $201 \mathrm{I}$. 
Por lo que, en este caso, se empleará la ecuación propuesta por el Centro de Investigaciones Sociales de Santiago de Chile, reportada por jiménez et al.I, para este tipo de estudios.

Para definir el tamaño de la muestra se utilizó la siguiente ecuación:

$$
n=\frac{P Q \times N}{(N-1) K_{2}^{\alpha_{2}}+P Q}
$$

Dónde:

$\mathrm{n}=$ Tamaño de la muestra

$P Q=$ Cuantil de la población $=0,5 \times 0,5=0,25$

$\mathrm{N}=$ Tamaño de la población $=8.427$ personas

$\alpha=\quad$ Intervalo o nivel de confianza $=0,05$

$\mathrm{K}=$ Constante de corrección del error $=2$

La muestra se seleccionó probabilísticamente, entre los miembros de la población, a la salida de las instalaciones del Gobierno Autónomo Descentralizado Municipal de Pangua, después de haber hecho o tratado de hacer algún trámite en la Institución. Al aplicar la formula arroja como resultado de muestra $38 \mathrm{I}$ personas encuestadas.

Las encuestas realizadas a los usuarios del GAD fueron diseñadas para complementar la información de la ejecución del presupuesto de acuerdo a lo planificado, nivel efectivo de autogestión, calidad en la prestación de los servicios municipales, costos de producción, insumos, tipo de siembra y cosecha, tipo de mano de obra, de esta forma se obtuvieron los datos requeridos para la investigación como la autogestión del gobierno autónomo repercute en la prestación de servicios.

Una vez aplicados los instrumentos, y obtenida la información necesaria, los datos fueron tabulados y cotejados mediante relación porcentual, para proceder a realizar el análisis respectivo. 


\section{RESULTADOS \\ Nivel efectivo de autogestión del Gobierno Autónomo Descentralizado Municipal de Pangua}

Uno de los aspectos que recogen estudios previos como elemento de mejora para los procesos de evaluación efectiva de la autogestión del gobierno autónomo descentralizado municipal del cantón Pangua repercute positivamente en la prestación de servicios. Para el caso de la muestra de estudio arrojó como resultado que una mayoría del $45 \%$ de los empleados/as encuestados, estiman que el nivel de autogestión es bueno.

Se analiza que una mayoría del $65 \%$ de los empleados encuestados, estiman que si cuentan con las facilidades para dar una atención de calidad en la prestación de los servicios municipales; el $35 \%$ de ellos consideran que no cuentan con las facilidades para dar una atención de calidad.

Según los datos, el $59 \%$ de los empleados encuestados conocen que si están realizando proyectos de autogestión para mejorar la prestación de los servicios municipales, y el $41 \%$ de restante dice que no están realizando proyectos.

\section{Nivel efectivo de autogestión en la prestación de los servicios municipales}

Según los datos el 33\% de los ciudadanos encuestados, estiman que el nivel de autogestión es deficiente; el $24 \%$ de ellos lo perciben como regular; el $20 \%$ como bueno; el I $2 \%$ muy bueno y ell I\% como excelente.

\section{Proyectos de autogestión realizados por el Gobierno Autónomo Descentralizado Municipal de Pangua}

Se analiza que una mayoría del $70 \%$ de los ciudadanos encuestados no conoce acerca de los proyectos de autogestión realizados por el Gobierno Autónomo Descentralizado Municipal de Pangua para mejorar la atención de los servicios municipales; el $30 \%$ dicen que si conocen.

\section{Participación en la elaboración del presupuesto del Gobierno Autónomo Descentralizado Municipal de Pangua}

Se analiza que una mayoría del $66 \%$ de los ciudadanos encuestados no han participado en la elaboración del presupuesto del Gobierno Autónomo Descentralizado Municipal de Pangua para mejorar la autogestión; el 34\% de ellos dicen que si han participado. Se analiza que una mayoría del $63 \%$ de los ciudadanos encuestados consideran que ha mejorado la calidad en la prestación de los servicios municipales en el Gobierno Autónomo Descentralizado Municipal de Pangua; el 37\% de ellos consideran que no.

Se analiza el 32\% de los ciudadanos encuestadosles gustaría un centro turístico que el Gobierno Autónomo Descentralizado Municipal de Pangua realice para el bienestar del cantón; un $25 \%$ les gustaría transporte municipal, el $20 \%$ agua potable; el I $2 \%$ alumbrado 
público; y el II\% otros proyectos como alcantarillado, hidroeléctrica.

\section{DISCUSIÓN}

Al momento de examinar: "Una evaluación efectiva de la autogestión del gobierno autónomo descentralizado municipal del cantón Pangua” repercute positivamente en la prestación de servicios, los empleados creen que si los evalúan periódicamente ellos se esfuerzan para dar un mejor servicio a la comunidad.

Los ciudadanos consideran que es deficiente el nivel de autogestión en el Gobierno autónomo descentralizado municipal de Pangua, y la mayoría de ellos no conocen acerca de proyectos de autogestión para mejorar la atención de los servicios municipales.

Las personas que fueron encuestadas en cuanto a los empleados la mayoría manifiestan que si se cumplen con la ejecución del presupuesto de acuerdo con lo planificado; pero la mayoría de ciudadanos encuestados no conocen y no han participado en la elaboración del presupuesto.

El aumento de la calidad en la atención incide positivamente en los servicios municipales, la mayoría de los empleados coincidieron que tienen todas las facilidades para dar una atención de calidad; el rendimiento laboral de una persona, está influenciado directamente por su estado de ánimo, por un cálido y equipado ambiente de trabajo, puesto que para hacer buenos proyectos, un buen informe, tener ganas de participar, el empleado debe estar tranquilo y contento; por el contrario si tienen preocupaciones, problemas familiares, peleas entre compañeros, la tristeza y ansiedad no dejarán espacio en su cerebro, para dar una buena atención al cliente. Los ciudadanos afirman que ha mejorado la calidad en la prestación de los servicios municipales

Una buena aplicación de estándares de calidad repercute positivamente en la atención de los servicios municipales, los empleados encuestados aseguró que los ciudadanos no los evalúan sobre la atención en la prestación de los servicios, situación que es confirmada por el $78 \%$ de ciudadanos, que manifestaron que no han calificado por ningún medio a los empleados del Gobierno autónomo descentralizado municipal de Pangua.

Los directivos entrevistados argumentaron que no tienen ningún sistema para que los ciudadanos evalúen a los empleados debido a que se les ha pasado por alto ese punto. Insisten que evaluar a los funcionarios sobre la calidad en la atención de los servicios municipales, ayudaría mucho a controlar sus impulsos y a responder de manera adecuada.

Los proyectos de autogestión con estos lograrían ser personas con una alta calidad y rapidez en los servicios hacia la comunidad .En cuanto a los ciudadanos el $82 \%$ desean 
que si realicen proyectos de autogestión ellos coincidieron con la teoría de Daniel Goleman (1995), la misma que conocieron durante la socialización que se realizó en primera instancia del proyecto.

\section{REFERENCIAS}

ARVON, Henry. (1982) "La participación autogestionaria". México: Editorial Zeta Bolsillo. $168 \mathrm{P}$

GÓMEZ BENÍTEZ, Armando. , 1983, Ponencia presentada durante el Seminario para la Formación de Capacitadores Municipales. Guadalajara, Jalisco.

PINCEMIN, Roberto. (1980) “La Autogestión”. Buenos Aires, Ediciones Román, 42p.

PINTO, M., GOMEZ, C., (2004) La cibera administración española en la sociedad de la información: retos y perspectivas, Gijón, Trea.

REBON, Julián., Saavedra Ignacio, (2006) “Empresas Recuperadas: La autogestión de los trabajadores". I Edición Buenos Aires. 109 p.

ROMERO, Aurora. (1992) “Investigación Participativa y Autogestión Comunitaria". Buenos Aires

VALLE, Ramón (1995)Gestión Estratégica de los Recursos Humanos Pearson Educación 